\title{
Supramolecular Catalysts Based on Novel Pyrimidinophane: Influence of Additives of Polymer and Lanthanum Ions
}

\author{
Dinar R. Gabdrakhmanov, ${ }^{@}$ Farida G. Valeeva, Vyacheslav E. Semenov, \\ Darya A. Samarkina, Anatoly S. Mikhailov, Vladimir S. Reznik, \\ and Lucia Ya. Zakharova
}

\author{
Dedicated to Academician of Russian Academy of Sciences O. G. Sinyashin \\ on the occasion of his $60^{\text {th }}$ Anniversary
}

\author{
A.E. Arbuzov Institute of Organic and Physical Chemistry, Kazan Scientific Center, Russian Academy of Sciences, 420088 \\ Kazan, Russian Federation \\ @Corresponding authorE-mail: Nemezc1988@yandex.ru
}

\begin{abstract}
Supramolecular systems based on the novel tetracationic amphiphilic pyrimidinophane, their mixtures with polymer (polyethyleneimine) and lanthanum ions were formed. Using spectrophotometry technique a catalytic activity of these systems was studied on the example of hydrolysis reaction of O-p-nitrophenyl-O-alkylchloromethylphosphonates (alkyl = ethyl (P1) and n-hexyl (P2)). At the first step the catalytic effect of individual solutions of pyrimidinophanes was examined. Pronounced substrate specificity for two different phosphonates was revealed: inhibition of hydrolysis for phosphonate with ethyl residue and catalysis of hydrolysis for phosphonate with n-hexyl moiety. Similar unusual effect for organized systems based on cationic surfactants could be a result of the influence of two opposite factors. On the one hand, $\mathrm{pH}$ decrease with the pyrimidinophane concentration was found. It corresponds to reducing of $\mathrm{OH}^{-}$ions concentration in reaction medium and, therefore inhibition of hydrolysis occurs. On the other hand, aggregation in aqueous solutions of pyrimidinophane induces reactant concentrating in surfactant aggregates that accelerates the reaction. Thereby depending on the domination of the first or the second factor inhibition or catalysis may be observed. Experimental kinetic data allow us to assume that phosphonate $\boldsymbol{P} 2$ because of its higher hydrophobicity and affinity to nonpolar core of micellar pseudophase possesses the higher capability to binding with pyrimidinophane aggregates in comparison with P1. Quantitative treatment of obtained kinetic data in terms of pseudophase model has revealed that binding ability of $\mathbf{P} \mathbf{2}$ with pyrimidinophane aggregates is almost 2 times greater than in the case of $\boldsymbol{P 1}$. Moreover, hydrolysis in catalytic complex proceeds more than 5 times faster for substrate with n-hexyl residue. This fact is also due to the high capability of $\mathbf{P} 2$ to concentrate within hydrophobic core of pyrimidinophane aggregates. Thus it can be concluded that in the case of $\mathbf{P} 2$ the domination of positive contribution over the negative contribution of $p H$ decrease with surfactant concentration is achieved due to high capability of substrate to bind with pyrimidinophane aggregates and high rate of hydrolysis in catalytic complex. It results in catalysis of reaction. At the same time, moderate ability of $\boldsymbol{P 1}$ to bind with aggregates is not enough to compensate pH decrease, which results in inhibition of reaction. Upon the transition from individual solutions of pyrimidinophane to its binary mixture with polyethyleneimine the difference of kinetic constants between $\boldsymbol{P} \mathbf{1}$ and $\boldsymbol{P} 2$ tends to be preserved. However absolute values of these constants are much lower than in the case of individual solutions of pyrimidinophane. Probably, it is due to the fact that cooperative aggregation between surfactant and polymer prevents reactant concentration in surfactant/polymer aggregates. However, resulting catalytic effect of binary system for both phosphonates is higher than in the case of individual solutions of pyrimidinophane. It is due to high contribution of general basic catalysis to summary effect with the participation of amino groups of PEI thereby levelling $\mathrm{pH}$ decrease. Addition of $\mathrm{La}^{3+}$ ions to the surfactant/polymer system allows of inverting catalytic activity from inhibition to catalysis accompanied by significant increase in kinetic constants for P1. Obviously it is due to electrophylic catalysis which involves $\mathrm{La}^{3+}$ ion coordination near oxygen atom of phosphoryl group. In the case of $\mathbf{P 2}$ expected increase in maximum of catalytic activity does not occur. Decrease in $\mathrm{OH}$-ions concentration during the formation of lanthanum hydroxocomplexes $[\mathrm{La}(\mathrm{OH})]^{2+}$ and $\left[\mathrm{La}(\mathrm{OH})_{2}\right]^{+}$due to salt hydrolysis could be the possible reason of this phenomenon. Thereby in the framework of this study supramolecular catalysts for cleavage of organophosphorous substrates were designed on the basis of novel amphiphilic tetracationic pyrimidinophane. Catalytic activity of the system could be controlled by addition of polymer and lanthanum salt. Results obtained could have practical relevance in terms of creation of effective agents for utilization of natural ecotoxicants.
\end{abstract}

Keywords: Pyrimidinophane, supramolecular catalysis, polyethyleneimine, substrate specificity. 


\title{
Супрамолекулярные катализаторы на основе нового пиримидинофана: влияние добавок полимера и ионов лантана
}

\author{
А. Р. Габдрахманов, ${ }^{@}$ Ф. Г. Валеева, В. Э. Семенов, А. А. Самаркина, \\ А. С. Михайлов, В. С. Резник, А. Я. Захарова
}

\section{Посвящается акаgемику Российской акаgемии наук О. Г. Синяшину в связи с его 60-летием}

Институт органической и физической химии им. А.Е. Арбузова Казанского научного иентра Российской академии наук, 420088 Казань, Россия

@E-mail: Nemezc1988@yandex.ru

\begin{abstract}
Сформированы супрамолекулярные системы на основе нового тетракатионного амфифильного пиримидинофана, его смесей с полимером (полиэтиленимин) и ионами лантана. Методом спектрофотометрии изучена каталитическая активность этих систем на примере реакиии гидролиза фосфонатов различной гидрофобности. Выявлена ярко выраженная субстратная специфичность: для более гидрофобного фосфоната наблюдается ускорение реакиии, величину которого можно контролировать добавками полимера и ионов металла, а в случае менее гидрофобного фосфоната происходит ингибирование гидролиза.
\end{abstract}

Ключевые слова: Пиримидинофан, супрамолекулярный катализ, полиэтиленимин, субстратная специфичность.

\section{Введение}

В настоящее время супрамолекулярная химия является мощным инструментом для создания полифункциональных систем. ${ }^{[1-7]}$ К числу ее наиболее интересных объектов относятся макроциклические соединения, практически полезные свойства которых связаны с возможностью инклюзивных взаимодействий по типу «гость-хозяин». ${ }^{[8-10]}$ В первую очередь, речь идет о каликсареновой платформе, механизмы функционирования которой построены на принципе молекулярного распознавания. ${ }^{[11-13]}$ Кроме этого, большое внимание в современной литературе уделяется комплексам на основе производных порфирина, которые хорошо зарекомендовали себя в качестве агентов для окисления различных углеводородов. ${ }^{[14-15]}$

Заслуживающей внимания стратегией модификации макроциклических соединений является введение в структуру различных функциональных групп и алкильных радикалов различной протяженности. ${ }^{[16-18]}$ Это наделяет систему способностью к самоорганизации, ${ }^{[19-20]}$ что позволяет качественно усовершенствовать функциональную активность систем. В частности, показана возможность использования функционали- зированной каликсареновой платформы для эффективного связывания и транспорта ДНК. ${ }^{[21]}$ На данный момент достигнуты определенные успехи в создании амфифильных макроциклических соединений, в основном производных порфирина, демонстрирующих ярко выраженные биомиметические свойства. ${ }^{[22-24]}$ В наших работах исследованы амфифильные макроциклы, содержащие пиримидиновый фрагмент (пиримидинофаны). ${ }^{[25]}$ Нами был разработан поэтапный дизайн полифункциональных систем путем перехода от индивидуального амфифила к его бинарной смеси с полимером и далее к тройной системе амфифил/полимер/ионы металла. Этот подход хорошо зарекомендовал себя для повышения активности супрамолекулярных катализаторов в реакции разложения фосфорорганических экотоксикантов (Рисунок 1). ${ }^{[26-27]}$ В рамках данной работы, выполненной в этом направлении, нами исследован тетракатионный пиримидинофан 1 (Рисунок 2a), его комплекс с полиэтиленимином (ПЭИ) (Рисунок 2б) и ионами $\mathrm{La}^{3+}$. Аналогично данным работы, ${ }^{[28]}$ можно ожидать, что увеличение числа заряженных центров и гидрофобности молекулы по сравнению с моно- и дикатионными аналогами, позволит значительно повысить каталитическую активность систем.

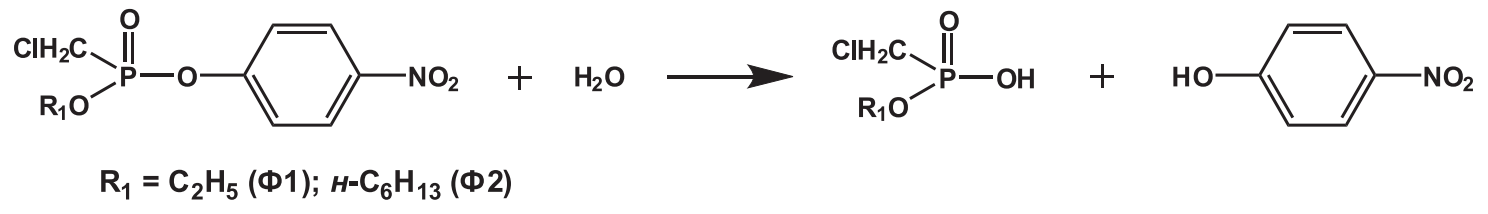

Рисунок 1. Схема гидролиза фосфонатов Ф1 и Ф2. 


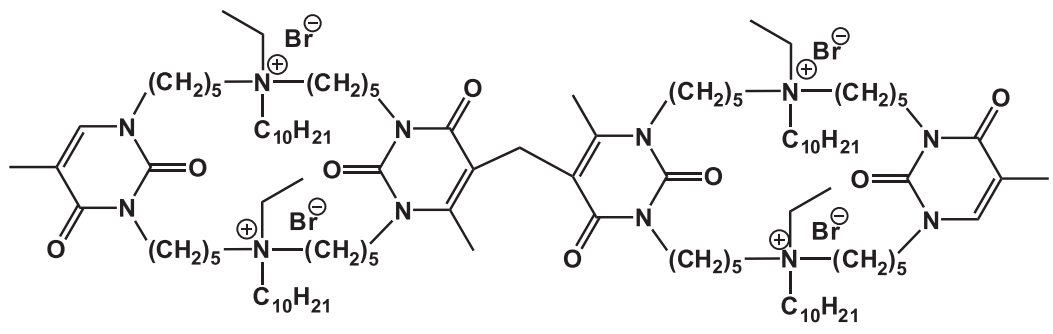

a<smiles>NCCNCCNCCN(CCNCCN(CCN)CCN)CCN(CCN)CCN(CCN)CCN</smiles>

6

Рисунок 2. Структура тетракатионного пиримидинофана 1 (а) и полиэтиленимина (б).

\section{Экспериментальная часть}

Пиримидинофан 1 получали согласно стандартной экспериментальной процедуре, детальное описание его синтеза сообщалось ранее. ${ }^{[25]} O$ - $n$-Нитрофенил- $O$ алкилхлорметилфосфонаты (алкил = этил (далее - фосфонат Ф1) и $н$-гексил (далее - фосфонат Ф2)) получали согласно методике. ${ }^{[29]}$

В качестве полимерной компоненты и соли использовали, соответственно, разветвленный полиэтиленимин (ПЭИ) (средняя молекулярная масса 50000, 50 \% водный раствор) и $\mathrm{La}\left(\mathrm{NO}_{3}\right)_{3} \cdot 6 \mathrm{H}_{2} \mathrm{O}$ («Aldrich»). Приведенные в работе мольные концентрации полимера рассчитаны на мономерное звено ПЭИ.

Спектрофотометрическое исследование кинетики гидролиза фосфонатов Ф1 и Ф2 проводили с применением спектрофотометра «Specord M-400», измеряя оптическую плотность $n$-нитрофенолят-аниона при 400 нм в условиях реакции псевдопервого порядка.

Для вычисления наблюдаемых констант скорости $\left(k_{\text {набл }}\right)$ использовали уравнение (1):

$$
\ln \left(A_{\infty}-A_{t}\right)=-k_{\text {набл }} t+\text { const, }
$$

где $A_{\infty}$ и $A_{t}$ - оптические плотности раствора по окончании реакции и в момент времени $t$, соответственно. Расчет осуществляли по методу взвешенных наименьших квадратов, во внимание принимали среднеарифметические значения результатов трех измерений, разница между которыми не превышала $5 \%$.

Количественный анализ кинетических данных проводили на основании уравнения (2), предполагающего формирование каталитического комплекса субстрат-мицелла: ${ }^{[30]}$

$$
k_{\text {набл }}=\frac{k_{w}+k_{\text {кат }} K_{S}^{\prime} C_{1}}{1+K_{S}^{\prime} C_{1}}
$$

где $k_{\text {набл }}-$ наблюдаемая константа скорости псевдопервого порядка; $k_{\text {кат }}$ и $k_{\mathrm{w}}-$ константы скорости первого порядка в каталитическом комплексе и в воде, соответственно; $K_{\mathrm{S}}$ (л/моль) приведенная константа связывания мицелл с субстратом; $C_{1}-$ концентрация пиримидинофана 1.

\section{Результаты и обсуждение}

На первом этапе была изучена каталитическая активность для индивидуальных растворов пиримидинофана 1. В ходе кинетического эксперимента была выявлена ярко выраженная субстратная специфичность: наблюдался переход от ингибирования реакции в случае менее гидрофобного фосфоната Ф1 к катализу для его более гидрофобного аналога Ф2 (Рисунок 3). Подобное явление для организованных систем на основе катионных ПАВ может быть следствием влияния двух противоположно направленных эффектов. С одной стороны, подобно ранее опубликованным данным, ${ }^{[25]}$ нами было выявлено снижение $\mathrm{pH}$ раствора с ростом концентрации пиримидинофана (Рисунок 3, вставка). Это эквивалентно снижению концентрации $\mathrm{OH}^{-}$-ионов в реакционной среде, что может явиться причиной ингибирования гидролиза. С другой стороны, самоорганизация пиримидинофана 1 в водных растворах вызывает концентрирование реагентов в агрегатах ПАВ, что увеличивает скорость протекания процесса. Таким образом, в зависимости от того, какой из этих двух факторов превалирует, может наблюдаться ингибирование или катализ гидролиза.

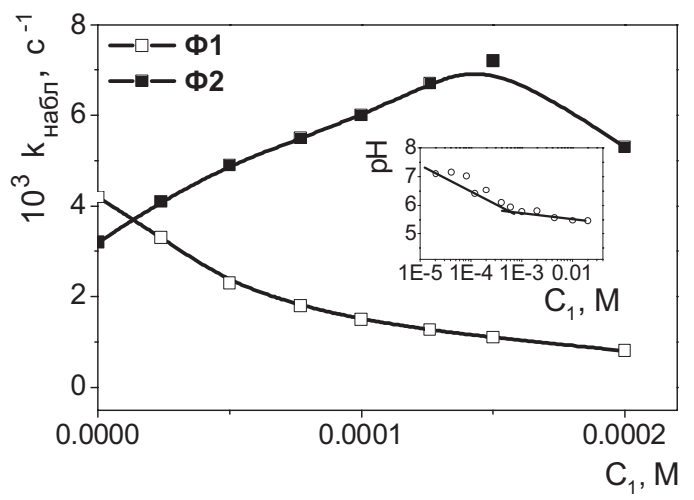

Рисунок 3. Зависимость наблюдаемой константы скорости щелочного гидролиза фосфонатов Ф1 и Ф2 от концентрации пиримидинофана $\mathbf{1}$ для индивидуальных растворов $\mathbf{1}$; $C_{\mathrm{NaOH}}=0.001 \mathrm{M} ; 25^{\circ} \mathrm{C}$. Вставка: зависимость $\mathrm{pH}$ водных растворов пиримидинофана 1 от его концентрации; $25^{\circ} \mathrm{C}$.

Экспериментальные кинетические данные позволяют предположить, что фосфонат $\mathbf{\Phi 2}$ по сравнению с $\mathbf{\Phi 1}$ в силу своей более высокой гидрофобности и сродства к неполярному ядру мицеллярной псевдофазы обладает более высокой способностью к связыванию с агрегатами пиримидинофана. Для проверки этой гипотезы проведена количественная обработка полученных результатов с использованием уравнения (2). Полученные данные приведены в Таблице 1. Действительно, более 
Таблица 1. Результаты количественной обработки кинетических данных с применением уравнения (2) для индивидуальных растворов пиримидинофана 1 , бинарной системы $1 /$ ПЭ и тройной системы $1 /$ ПЭИ/ $\mathrm{La}^{3+}$.

\begin{tabular}{|c|c|c|c|c|}
\hline Система & Фосфонат & $k_{\text {кат }} \times 10^{3}, \mathrm{c}^{-1}$ & $K_{\mathrm{S}}^{\prime}$, л/моль & $\left(k_{\text {набл }} / k_{\mathrm{w}}\right)_{\max }$ \\
\hline \multirow[t]{2}{*}{1} & $\Phi 1$ & 1.2 & 8898 & 0.2 \\
\hline & $\Phi 2$ & 6.3 & 15793 & 2.3 \\
\hline \multirow[t]{2}{*}{ 1/ПЭИ } & $\Phi 1$ & 0.2 & 384 & 0.5 \\
\hline & $\Phi 2$ & 5.5 & 4570 & 6.8 \\
\hline \multirow[t]{2}{*}{ 1/ПЭИ/La ${ }^{3+}$} & $\Phi 1$ & 5.5 & 1778 & 2.0 \\
\hline & $\Phi 2$ & 32.0 & 4084 & 7.3 \\
\hline
\end{tabular}

гидрофобный фосфонат $\mathbf{\Phi 2}$ способен практически в 2 раза эффективнее связываться с агрегатами $\mathbf{1}$ по сравнению с Ф1 (Таблица 1). Кроме этого, в случае субстрата с $\mu$-гексильным радикалом гидролиз в каталитическом комплексе протекает более чем в 5 раз быстрее (Таблица 1), что также связано с его более высокой способностью концентрироваться в гидрофобном ядре агрегатов пиримидинофана.

Таким образом, в случае $\mathbf{\Phi 2}$ достигается доминирование положительного вклада факторов мицеллярного катализа над отрицательным эффектом снижения $\mathrm{pH}$ системы, что в результате приводит к катализу реакции. В то же время, способности Ф1 к связыванию с агрегатами ПАВ недостаточно для того, чтобы компенсировать ингибирование реакции вследствие понижения $\mathrm{pH}$ раствора с ростом концентрации $\mathbf{1}$, что в результате приводит к общему ингибированию процесса.

При переходе от индивидуальных растворов 1 к его бинарной смеси с ПЭИ (Рисунок 4) сохраняются те же различия констант скорости субстратов $\mathbf{\Phi 1}$ и $\mathbf{\Phi 2}$, что и в случае индивидуального ПАВ (Таблица 1). Однако абсолютные значения констант связывания субстратов с агрегатами ПАВ и констант скорости уменьшаются. Вероятно, включение полимера в агрегаты затрудняет доступ субстрата к пиримидинофану и препятствует концентрированию реагентов в смешанных агрегатах ПАВ/полимер. Тем не менее, результирующий каталитический эффект бинарной системы для обоих фосфонатов выше, чем в случае индивидуальных растворов 1 (Таблица 1). Этот факт можно объяснить дополнительным вкладом в суммарный каталитический эффект общего основного

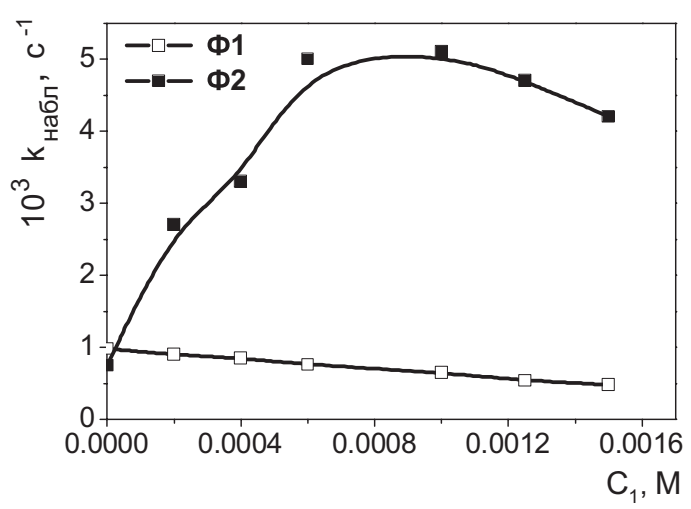

Рисунок 4. Зависимость наблюдаемой константы скорости гидролиза фосфонатов $\mathbf{\Phi 1}$ и $\mathbf{\Phi 2}$ от концентрации 1 для бинарной системы $1 /$ ПЭИ; $C_{\text {пэи }}=0.05 \mathrm{M} ; 25^{\circ} \mathrm{C}$. катализа с участием аминогрупп ПЭИ, нивелирующим эффект снижения $\mathrm{p} H .^{[25]}$

Известно, что ионы металлов оказывают каталитическое действие на нуклеофильное замещение в эфиpax кислот фосфора даже в отсутствие лигандов. ${ }^{[31-33]}$ При этом в литературе рассматривается комплексный механизм действия, включающий поляризацию фосфорильного фрагмента, усиление нуклеофильных свойств воды (снижение $\mathrm{p} K_{\mathrm{a}}$ ), перенос нуклеофила к реакционному центру и стабилизацию аниона уходящей группы. В мицеллярных средах наряду с гомогенно каталитическим механизмом реализуется значительный вклад в суммарный эффект, связанный с изменением мицеллообразующих характеристик ПАВ. Ранее было показано высокое каталитическое действие ионов лантана, превосходящее влияние других солей переходных металлов и лантаноидов. ${ }^{[33]}$ В водных средах происходит образование гидроксокомплексов лантана состава $[\mathrm{La}(\mathrm{OH})]^{2+}$ и $\left[\mathrm{La}(\mathrm{OH})_{2}\right]^{+}$, способных к координированию по атому кислорода фосфорильной группы, что в значительной степени увеличивает электрофильность атома фосфора.

Введение в систему ионов $\mathrm{La}^{3+}$ значительно увеличивает каталитическую активность в случае фосфоната Ф1 с инверсией каталитического эффекта от ингибирования к катализу (Рисунок 5). Очевидно, в данном случае имеет место дополнительный вклад электрофильного катализа с участием ионов $\mathrm{La}^{3+}$. Для фосфоната Ф2 по этой же причине наблюдается резкое повышение величины $k_{\text {кат }}$ однако ожидаемого значительного роста максимального каталитического эффекта (величина $K$, изменяется незначительно) не происходит (Таблица 1).

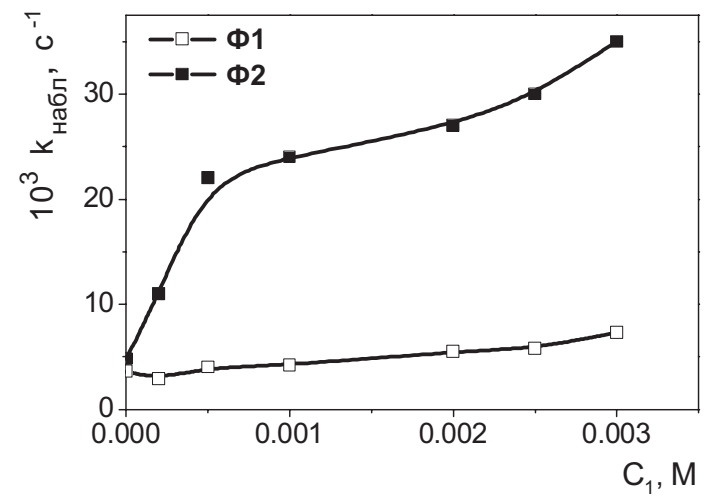

Рисунок 5. Зависимость наблюдаемой константы скорости гидролиза фосфонатов $\boldsymbol{\Phi} 1$ и $\boldsymbol{\Phi 2}$ от концентрации 1 для тройной системы $1 /$ ПЭИ/ $\mathrm{La}^{3+} ; C_{\text {пэи }}=0.05 \mathrm{M} ; C_{\mathrm{La}^{3+}}=0.008 \mathrm{M} ; 25^{\circ} \mathrm{C}$. 
Возможной причиной подобного явления может быть понижение концентрации $\mathrm{OH}^{-}-$-ионов в ходе образования вышеупомянутых гидроксокомплексов лантана.

\section{Выводы}

Таким образом, в рамках данной работы методом поэтапного дизайна созданы супрамолекулярные катализаторы на основе нового амфифильного пиримидинофана для разложения эфиров кислот фосфора, каталитическую активность которых можно регулировать путем добавок полимера и неорганической соли. Полученные результаты могут иметь практическую значимость с точки зрения создания эффективных агентов для утилизации фосфорорганических экотоксикантов.

Благодарности. Работа выполнена при финансовой поддержке Российского научного фонда (проект № 1450-00014).

\section{Список литературы}

\section{References}

1. Raynal M., Ballester P., Vidal-Ferran A., Van Leeuwen P.W.N.M. Chem. Soc. Rev. 2014, 43, 1160-1733.

2. Di Stefano S., Cacciapaglia R., Mandolini L. Eur. J. Org. Chem. 2014, 33, 7304-7315.

3. Brown C.J., Toste F.D., Bergman R.G., Raymond K.N. Chem. Rev. 2015, 115, 3012-3035.

4. Gaynanova G.A., Vagapova G.I., Valeeva F.G., Vasilieva E.A., Galkina I.V., Zakharova L.Ya., Sinyashin O.G. Colloids Surf. A 2015, 489, 95-102.

5. Gabdrakhmanov D.R., Samarkina D.A., Valeeva F.G., Saifina L.F., Semenov V.E, Reznik V.S., Zakharova L.Ya., Konovalov A.I. Russ. Chem. Bull. 2015, 64, 573-578.

6. Mukhametshina A., Mustafina A., Syakaev V., Amirov R., Petrov K., Konovalov A. J. Mol. Liq. 2015, 211, 839-845.

7. Kataev E.A., Müller C. Tetrahedron 2014, 70, 137-167.

8. Ouyang G.-H., He Y.-M., Li Y., Xiang J.-F., Fan Q.-H. Angew. Chem. Int. Ed. 2015, 54, 4334-4337.

9. Taylor M.S. Acc. Chem. Res. 2015, 48, 295-305.

10. Yin Y., Jiao S., Lang C., Liu J. RSC Adv. 2014, 4, 25040-25050.

11. Bistri O., Reinaud O. Org. Biomol. Chem. 2015, 13, 28492865.

12. Tan R.H. Adv. Mater. Res. 2014, 887-888, 647-650.

13. Winner L., Daniloff G., Nichiporuk R.V., Solovyov A., Katz A. Top. Catal. 2015, 58, 441-450.
14. García-Simón C., Gramage-Doria R., Raoufmoghaddam S., Parella T., Costas M., Ribas X., Reek J.N.H. J. Am. Chem. Soc. 2015, 137, 2680-2687.

15. Huang G., Mo L.-Q., Cai J.-L., Cao X., Peng Y., Guo Y.-A., Wei S.-J. Appl. Catal. B 2015, 162, 364-371.

16. Morozova J.E., Syakaev V.V., Ermakova A.M., Shalaeva Y.V., Kazakova E.K., Konovalov A.I., Colloids Surf. A 2015, 481, 400-406.

17. Wang K.-P., Chen Y., Liu Y. Chem. Commun. 2015, 51, 16471649.

18. Mironova D.A., Muslinkina L.A., Morozova J.E., Shalaeva Y.V., Kazakova E.K., Kadyrov M.T., Nizameev I.R., Konovalov A.I. Colloids Surf. A 2015, 468, 339-345.

19. Basilio N., Francisco V., Garcia-Rio L. Int. J. Mol. Sci. 2013, 14, 3140-3157.

20. Rodler F., Schade B., Backes S., Hampel F., Böttcher C., Clark T., Hirsch A. J. Am. Chem. Soc. 2015, 137, 3308-3317.

21. Rodik R.V., Anthony A.-S., Kalchenko V.I., Mély Y., Klymchenko A.S. New J. Chem. 2015, 39, 1654-1664.

22. Zhu S., Wu F., Wang K., Zheng Y., Li Z., Zhang X., Wong W.K. Bioorg. Med. Chem. Lett. 2015, 25, 4513-4517.

23. Li H., Chan C.-F., Chan W.-L., Lear S., Cobb S.L., Mak N.-K., Lau T.C.-K., Lan R., Wong W.-K., Wong K.-L. Org. Biomol. Chem. 2014, 12, 5876-5882.

24. Tovmasyan A., Babayan N., Poghosyan D., Margaryan K., Harutyunyan B., Grigoryan R., Sarkisyan N., Spasojevic I., Mamyan S., Sahakyan L., Aroutiounian R., Ghazaryan R., Gasparyan G. J. Inorg. Biochem. 2014, 140, 94-103.

25. Zakharova L.Ya., Semenov V.E., Syakaev V.V., Voronin M.A., Gabdrakhmanov D.R., Valeeva F.G., Mikhailov A.S., Voloshina A.D., Reznik V.S., Latypov Sh.K., Konovalov A.I. Mater. Sci. Eng. C 2014, 38, 143-150.

26. Zakharova L.Ya., Semenov V.E., Voronin M.A. Valeeva F.G., Ibragimova A.R., Giniatullin R.Kh., Chernova A.V., Kharlamov S.V., Kudryavtseva L.A., Latypov Sh.K., Reznik V.S., Konovalov A.I. J. Phys. Chem. B 2007, 111, 1415214162.

27. Voronin M.A., Valeeva, F.G., Zakharova L.Ya., Giniyatullin R.Kh., Semenov V.E., Reznik V.S. Kinet. Catal. 2010, 51, 644-652.

28. Zakharova L.Ya., Voronin M.A., Semenov V.E., Gabdrakhmanov D.R., Syakaev V.V., Gogolev Yu.V., Giniyatullin R.Kh., Lukashenko S.S., Reznik V.S., Latypov Sh.K., Konovalov A.I., Zuev Yu.F. ChemPhysChem 2012, 13, 788-796.

29. Patent USA 2922810, 1960.

30. Berezin I.V., Martinek K., Yatsimirski A.K. Uspekhi Khimii 1973, 42, 1729-1756.

31. Jenks V. Catalysis in Chemistry and Enzymology, Moscow: Mir, 1972. 468 p. (transl. into Russ.) [Дженкс В. Катализ в химии и энзимологии, М.: Мир, 1972. 468 с.].

32. Tsubouchi A., Bruice T.C. J. Am. Chem Soc. 1995, 117, 73997411.

33. Zakharova L.Y., Valeeva F.G., Kudryavtseva L.A., Konovalov A.I. Mendeleev Commun. 2000, 10, 241-243. 